\title{
Orientational ordering and spatial distribution of Janus nanoparticles in lamellae diblock copolymers
}

\author{
M. A. Osipov ${ }^{1,2}$ and A. S. Ushakova ${ }^{2}$ \\ 1 Department of Mathematics and Statistics, \\ University of Strathclyde, Glasgow G1 1 XH, Scotland, UK \\ 2 Topchiev Institute of Petrochemical Synthesis, \\ Russian Academy of Sciences, 119991 Moscow, Russia
}

\begin{abstract}
Orientational ordering and spatial distribution of rod-like Janus nanoparticles in the lamellae diblock copolymers has been considered using a molecular theory based on simple model of Janus particle with two different interaction centres located at the particle ends. Order parameter and concentration profiles of Janus nanoparticles have been calculated numerically for different values of the nanoparticle length. It has been found that Janus nanoparticles are mainly located in the boundary region between the domains and are orientationally ordered in that region with a high orientational order parameter. This orientational order is induced by the interface and is determined by the different affinities of the two ends of the Janus particle with respect to the monomers of different domains. Comparison with block copolymers doped with symmetric nanoparicles indicates that polymer nanocomposites with Janus particles are expected to possess much larger dielectric abisotropy and thus can be considered as promising anisotropic nanomaterials which can be aligned by the external electric field.
\end{abstract}




\section{INTRODUCTION}

At present significant attention is attracted to soft matter nanocomposites based on anisotropic molecular liquids doped by metal, dielectric and semiconductor nanoparticles. Variuos properties of such materials, including thermodynamical,optical nad dielectric ones,may be modified by the presence of even a small fraction of nanoparticles [1] In particular, nematic liquid crystals doped with few volume percent of various nnaoparticles are charaxterised by a number of improved characteristics including an improvement of such important parameters of liquid crystal displays as switching voltages and switching times. (see [2-6]).Another example is an increase of the temperature range of liquid crystal blue phases [7] by using a small admixture of metal nanoparticles. Structure and properties of smectic liquid crystals may also be modified by nanoparticles. For example, semiconductor quantum dots may positionally order in side chain liquid crystal polymers $[8,9]$ resulting in the formation of a self-assembled soft nanocomposite.

A pioneering study of the effect of ferroelectric nanoparticles on the phase transitions and dielectric properties of liquid crystals has been undertaken by Yu. Reznikov and his group $[11,12,14,15]$ It has been shown that even a very small molar fraction of strongly polar ferroelectric NPs (of the order of $10^{-3}$ ) results in a dramatic increase of the dielectric constant of the composite which is of the same order as the dielectric constant of the host nematic phase itself $[11,15]$. It has also been shown that the nematic-isotropic transition temperature is significantly shifted by ferroelectric nanoparticles $[11,12]$. These studies of Reznikov et.al. have been extended by other authors who have shown also that doping of nematics with ferroelectric nanoparticles leads to an increase of their electro-optic response $[16,17]$

Other types of nanoparticles may also effect the thermodynamic stability of liquid crystal phases due to their orientational ordering in the anisotropic host phase. For example, it has been verified experimentally that the nematic-isotropic (N-I) phase transition temperature is decreased when the nematic liquid crystal is doped with isotropic nanoparticles inluding silver [18], gold [19] or aerosil ones [20,21]. On the other hand, an increase of the N-I transition temperature is observed when the nematic liquid crystal is doped with strongly anisotropic particles including nanotubes [22], magnetic nanorods [10] and ferroelectric nanoparticles $[11,12]$. These effects have recently been described theoretically [13, 23]. 
Nanoparticles of various chemical structure may also significantly improve mechanical, elctrical and optical characteristics of block copolymers [24-30]. However, from the standpoint of liquid crystal science the most interesting effects are related to the orinetational ordering of anisotropic nanoparticles in block copolymers. One notes that both hexagonal and lamellae block copolymers are uniaxial, and thus, in principle, sufficiently anisotropic nanorods should possess some degree of induced orientational order in the corresponding composites. In the lamellae phase the symmetry axis is in the direction perpendicular to the flat boundaries between the blocks while in the hexagonal phase the axis is parallel to that of the cylindrical block. On the other hand, the physical anisotropy of diblock copolymers is relatively weak because the material in the bulk of each block is isotropic and the macroscopic anisotropy is only determined by the boundaries. Thus sufficiently small anisotropic nanoparticles are expected to order orientationally only in the interfacial regions between the adjacent blocks where the nanoparticles interact with the monomers of both blocks. Nanoparticles interact differently with the monomers of the two different kinds and this results in the effective anisotropic mean-field in the boundary region which aligns the nanoparticles [35].If the length of the nanorod is comparable with the size of the block, one expects some orientational ordering throughout the system becuase a given nanorod always interacts with at least one block boundary. The orientational ordering of nanorods in diblock copolymers has indeed been observed experimentally. Metal nanorods with various functional groups are aligned parallel to the domain walls in the lamellae [31] and to the cylindrical domain walls in the hexagonal phase [34]. In contrast, polystyrene functionalised nanorods are ordered in the hexagonal phase perpendicular to the cylinders [32, 33].

Recently spacial distribution of anisotropic nanoparticles and their orientational order in the lamellae and hexagonal phases of diblock copolymers has been studied in detail theoretically [35] assuming the fixed phase-separated structure of the copolymer. In this approximation the theory has much in coomon with the molecular theory of nematic liquid crystals in the external field as the orientational order of nanoparticles in induced by the effective mean filed created by the average interaction between the nanoparticles and the monomers of both blocks. In that theory a nanoparticle is assumed to be spherical but, at the same time, it possesses some anisotropic properties (for example, anisotropic polarizability or the quadrupole momemnt). As a result the potential of interaction between a nanoparticle and a monomer is composed of the isotropic and the anisotropic parts Here 
the anisotropic model potential, which apparently has not been considered before in the theory of polymers, is determined by effective particle anisotropy. Orientational order parameter profiles of nanoparticles have been calculated numerically and it has been shown that anisotropic nanoparticles are orientationally ordered in the interfacial region. The most interesting result obtained in [35] is tht the nanoparticles are aligned parallel to the domain wall in one of the blocks and perpendicular to the wall in the adjacent block.

The main results of Ref. [35] have recently been verified bv molecular dynamics simulation [36] of the spatial distribution and orientational ordering of nanorods in the lamellar block copolymer phase. Anisotropic nanoparticles have been modelled by a rigid string of spheres interacting with the monomers of both blocks, and dissipative particle dynamics has been used to evaluate concentration distribution of both monomers and the nanoparticles and the orientational order parameter profiles for different values of the nanoparticle length and selectivity The simulations confirm the sign inversion of the nematic order parameter at the domain wall but, in contrast to the molecular theory, the nanoparticles are ordered both in the boundary region and in the bulk of the domains and the nematic order parameter, extracted from simulations, appears to be higher than in theory. These discrepancies are related to the large geometrical anisotropy of the nanoparticles used in simulations which is of the same order as the period of the simulated lamellae structure. At the same time in the molecular theory the nanoparticles are assumed to be spherical.

In our recent paper [37] the agreement between theory and computer simulations has been significantly improved by using a different model of a nanoparticle which is composed of two equal spherical interaction cites separated by a distance $l$. Similar to the nanoparticles employed in the computer simulations, the interaction between such a model particle and a monomer is not central and explicitly depends on the nanoparticle length. The molecular theory based on such a model yields sufficiently high values of the orientational order parameter and the anisometric nanoparticles are orintationally ordered in a broader interfacial region. However, from the application point of view, both composites considered theoretically in [35] and [37] have a common disadvantage. In both cases the nanoparticles are predominantly located in one of the blocks and as a result the fraction of nanoparticles, which are orientationslly ordered in the boundary region, appear to be rather small. Hence the average macroscopic anisotropy of such a composite is expected to be small. At the same time one of the most important potential chracteristics of polymer nanocomposite materi- 
als with orientationally ordered nanoparticles is the sufficiently large dielectric anisotropy which, in principle, enables one to align block copolymer nanocomposites using external electric field. At present the alignment of block copolymers is a complicated problem which is far from being completely solved.

In this paper we consider the orientational ordering and spatial distribution of the so called Janus nanoparticles in the ideal lamellae phase. Janus particles are composed of two different spherical interaction cites separated by the distance $l$. One of the spheres interacts stronger with the monomers of the block $\mathrm{A}$ and hence it has a preference to be located in the block A. At the same time the second sphere has the same preference to be located in the block B. One expects that Janus particles are predominantly located in the boundary region between the blocks where each sphere may reside in the corresponding preferable domain. In contract, the energy of such a nanoparticle is rather high when it is located inside the block and hence the concentration of Janus nanoparticles inn the bulk of both domains should be sufficiently small. The paper is arranged as follows. In Section 2 we summarise the results of the molecular field theory of polymer nanocomposites and derive explicit expressions for the nematic order parameter and the concentration distribution of Janus nanoparticles in the strongly segregated lamellae phase of a diblock copolymer.In Section 3 we present our numerical results and Section 4 contains a detailed discussion.

\section{MOLECULAR THEORY OF DIBLOCK COPOLYMER NANOCOMPOSITES WITH JANUS NANOPARTICLES}

In this paper we consider a simple model of a Janus nanoparticle which is composed of two spheres of radius $r_{0}$ separated by the distance $L$ (see Fig. 1). The spheres have the same radius but have a different affinity with the monomers $\mathrm{A}$ and $\mathrm{B}$ of the corresponding blocks of a diblock copolymer. The interaction potential between the sphere $i$ of a nanoparticle $(i=1,2)$ and the monomer $j$ of the kind $\alpha(\alpha=A, B)$ is given by a simple expression $V\left(r_{i j}\right)=J_{i, \alpha} r_{i j}^{-6}$ with the steric cut-off at $r_{i j}=r_{0}$, i.e. $V\left(r_{i j}=0\right.$ if $r_{i j}<r_{0}$. Here $J_{i, \alpha}$ is the coupling constant which generally depends both on the type of monomer $\alpha$ and the type of the sphere $i$ in the asymmetric Janus nanoparticle. Thus the total interaction potential 
between a nanoparticle $p$ and all monomers in the blockcopolymer is expressed as:

$$
V_{p}=\sum_{l=l_{A}}\left[J_{1, A}\left(r_{1 p l}\right)+J_{2, A}\left(r_{1 p l}\right)\right]+\sum_{l=l_{B}}\left[J_{1, B}\left(r_{2 p l}\right)+J_{2, B}\left(r_{2 p l}\right)\right]
$$

where $\mathbf{r}_{1 p j}=\mathbf{r}_{p j}+\left(L+r_{0}\right) \mathbf{a}_{p}, \mathbf{r}_{2 p j}=\mathbf{r}_{p j}-\left(L+r_{0}\right) \mathbf{a}_{p}$ and where $\mathbf{r}_{p j}$ is the vector between the centre of the nanoparticle $p$ and the monomer $j$. Here the unit vector $\mathbf{a}_{p}$ is along the linear nanoparticle.

We assume that the sphere "1" interacts stronger with the monomers A and hence $J_{1, A}-$ $J_{1, B}=\Delta J_{1}<0$. In contrast, for sphere " $2 " J_{2, A}-J_{2, B}=\Delta J_{2}>0$. For simplicity we assume that $\Delta J_{1}=-\Delta J_{2}=\Delta J$.

In the molecular field approximation, the single NP spatial and orientational distribution function is given by the Boltzman expression:

$$
f\left(\mathbf{a}_{p}, \mathbf{r}_{p}\right)=Z^{-1} \exp \left[-U_{M F}\left(\mathbf{a}_{p}, \mathbf{r}_{p}\right) / k_{B} T\right]
$$

where $Z$ is the normalization factor and the mean-field potential $U_{M F}\left(\mathbf{a}_{i}, \mathbf{r}_{i}\right)$ is expressed as:

$$
\begin{aligned}
& U_{M F}\left(\mathbf{a}_{p}, \mathbf{r}_{p}\right)= \\
& \int\left[J_{1, A}\left(r_{p l}\right)+J_{2, A}\left(r_{p l}\right)\right] \rho_{A}\left(\mathbf{r}_{l}\right) d^{3} \mathbf{r}_{l}+ \\
& \int\left[J_{1, B}\left(r_{p l}\right)+J_{2, B}\left(r_{p l}\right)\right] \rho_{B}\left(\mathbf{r}_{l}\right) d^{3} \mathbf{r}_{l},
\end{aligned}
$$

where $\rho_{\alpha}\left(\mathbf{r}_{l}\right)$ is the density distribution of the monomers $\alpha$. In the case of strong segregation $\rho_{\alpha}(\mathbf{r})=\rho_{0, \alpha}$ when $0<z<d_{\alpha}, \rho_{\alpha}(\mathbf{r})=0$ when $d_{\alpha}<z<d$, and $\rho_{\alpha}(z)=\rho_{\alpha}(z+d)$ for all $z$ as it is a periodic function with the period $d$. Here $d_{\alpha}$ is the thickness of the block $\alpha$, the axis $z$ is perpendicular to the lamellae planes and . $\rho_{0, \alpha}$ is the average density of monomers $\alpha$ in the block $\alpha$.

One notes that the mean-field potential is a sum of the potentials for the two spheres of the Janus particle. For a single interaction sphere it is possible to obtain an explicit analytical expression for the mean-field potential Ref. [35], which is given by the following expressions in different ranges of $z$ where $z$ is the distance from the nanoparticle to the domain wall. Assuming that the range of interaction is much larger then $r_{0}$, the mean field potential for one sphere canbe written in the form: 
i) For $z>r_{0}$

$$
U_{M F i}(z)=\frac{\pi}{6} \Delta J z^{-3}-\frac{2 \pi}{3} \Delta J r_{0}^{-3}
$$

ii) for $0<z<r_{0}$

$$
U_{M F i}(z)=-\frac{\pi z}{2} \Delta J r_{0}^{-4}
$$

The lamellae phase is uniaxial and hence the mean-field potential depends only on $z$ and $\cos \theta$ where $\theta$ is the angle between the nanoparticle axis and the $z$ axis. The total mean-field potential for the nanoparticle $p$ can now be written as a sum of two terms:

$$
U_{M F}(z, \theta)=U_{M F 1}\left(z_{1}\right)+U_{M F 2}\left(z_{2}\right)
$$

where $z_{1}=z+\left(L / 2+r_{0}\right) \cos \theta$ is the distance from the sphere "1" to the flat interface and $z_{2}=z-\left(L / 2+r_{0}\right) \cos \theta$ is the distance from the sphere " $2 "$ and where both terms in Eq.() are given by Eq. () with $z=z_{1}$ and $z=z_{2}$ respectively.

One notes that the particular expression for the total mean-field potential of a Janus nanoparticle depends on the inequalities $\left|z_{1}\right|<r_{0}$ and $\left|z_{2}\right|<r_{0}$, which are satisfied in different rages of $z$ and $\theta$ because $z_{1}$ and $z_{2}$ depend both on $z$ and $\theta$. As a result the plane $(z, \cos \theta)$ is into many regions and the integration should be undertaken separately within each region using the variable limits. Such a procedure is cumbersome and computationally challenging. On the other hand, the numerical integration can be dramatically simplified by using the following interpolated mean-field potential [37] which is qualitatively valid in the whole range of $z$ and $\theta$ :

$$
\begin{aligned}
U_{M F}^{*}(z, \theta)=-\frac{\pi}{6} \Delta J \tanh \left(z_{1}^{6} / r_{0}^{6}\right) z_{1}^{-3} & -\frac{2 \pi}{3} \Delta J \tanh \left(z_{1} / r_{0}\right) r_{0}^{-3} \\
& +\frac{\pi}{6} \Delta J \tanh \left(z_{2}^{6} / r_{0}^{6}\right) z_{2}^{-3}+\frac{2 \pi}{3} \Delta J \tanh \left(z_{2} / r_{0}\right) r_{0}^{-3},
\end{aligned}
$$

where $\tanh \left(z / r_{0}\right)$ and $\tanh \left(z^{6} / r_{0}^{6}\right)$ are the interpolation functions. For $z>r_{0} \tanh \left(z / r_{0}\right) \approx$ $\tanh \left(z^{6} / r_{0}^{6}\right) \approx 1$ and for $z<r_{0} \tanh \left(z / r_{0}\right) \approx z / r_{0}$ and the first term proportional to $\tanh \left(z_{2}^{6} / r_{0}^{6}\right) \approx z^{6} / r_{0}^{6} \ll 1$ can be neglected.

The interpolated potential $U_{M F}^{*}(z, \theta)$ is shown in Fig.2 for two different values of $\theta=\pi / 3$ and $\theta=\pi / 4$. One notes that the potential is symemtric with respect to the domain wal and possesses a minimum at the boundary. 
Now the local orientational order parameter $S(\mathbf{r})$ of the anisotropic nanoparticles can be written in the form:

$$
S(\mathbf{r})=\left\langle P_{2}\left(\mathbf{a}_{p} \cdot \mathbf{k}\right)\right\rangle=\frac{\int P_{2}\left(\mathbf{a}_{p} \cdot \mathbf{k}\right) f\left(\mathbf{a}_{p}, \mathbf{r}\right) d^{2} \mathbf{a}_{p}}{\int f\left(\mathbf{a}_{p}, \mathbf{r}\right) d^{2} \mathbf{a}_{p}}
$$

where the unit vector $\mathbf{k}$ is in the direction of the $z$ axis and $P_{2}(x)$ is the second Legendre polynomial..

The density distribution of nanoparticles is given by

$$
\rho_{N}(\mathbf{r})=\rho_{N 0} \int f\left(\mathbf{a}_{i}, \mathbf{r}\right) d^{2} \mathbf{a}_{i}
$$

where $\rho_{N 0}$ is the average number density of nanoparticles.

\section{ORDER PARAMETER AND DENSITY PROFILES OF JANUS NANOPAR- TICLES}

Now the orientational order parameter $S(z)$ and the local concentration of nanoparticles can be calculated numerically substituting the mean-field potential (7) into Eqs. (8) and (9). The corresponding profiles for relatively short Janus nanoparticles are presented in Figs. 3 and 4. For comparison in Figs. 3 and 4 we also present the order parameter and concentration distributions for symmetric nanoparticles of the same length which are composed of two equal speheres which interact with monomers A and B exactly in the same way as one of the spheres of the asymmetric Janus particles. One can readily see in Fig.4 that Janus nanoparticles are predominantly located in a boundary region between the adjacent blocks. This is related to the shape of the corresponding mean-field potential which has a pronounced minimum at the boundary. In contrast, symmetric nanoparticles are mainly located in the bulk of one of the domains because they have an affinity to the monomers of the corresponding type.

Both symmetric nanoparticles and Janus nanoparticles are orientationally ordered in the boundary region of approximately the same length as shown in Fig.3. However, the orientational order parameter profiles are completely different. Local nematic order parameter of Janus nanoparticles reaches its maxima (with $S \approx 0.6$ ) symmetrically on both sides of the domain wall. Thus the Janus particles are strongly orientationally ordered when the nanoparticle centre is separated by approximately $2 r_{0}$ from the boundary. At the same time 
the orientational order parameter is much smaller $(S \approx 0.1)$ if the nanoparticle centre is located directly on the boundary. This can be explained qualitatively in the following way. Consider the Janus nanoparticle with its centre located at the boundary. In this case the minimum of the interaction energy is achieved when each sphere resides in the favorable domain (see Fig.1). The interaction energy, however, does not depend much on the angle $\theta$ between the nanoparticle axis and the boundary normal until the spheres touch the boundary. Thus in this geometry the orientational distribution function is shallow and the order parameter is small. In contrast, if the distance between the Janus nanoparticle and the boundary is approximately half the nanoparticle length, both spheres may still be located in their favorable domains but the interaction energy now strongly depends on the angle $\theta$. Indeed, after even a small rotation of the Janus nanoparticle one of the spheres may leave its favorable domain and cross the wall which will lead to a significant increase of the energy. Thus the nanoparticles are strongly orientationally ordered in this case.

Symmetric nanoparticles also possess strong orientational order in the boundary region but their orientational order parameter $S$ changes sign at the boundary. This sign reversal can be explained in the following way [37]. Let us consider a symmetric nnaoparticle located sufficiently close to the boundary. If the nanoparticle centre is located in the block A where the nanoparticles interact stronger with monomers then in Block B, it is enenrgetically favorable for the symmetric nanoparticle to reside in Block A. However, this is only possible if the nanoparticle is approximately parallel to the boundary between the blocks. In contrast, if the NP centre is located in the block B, the two spheres cannot be located in the block A simultaneously, and the minimum of the interaction energy is achieved when one of the two spheres sphere is in the block A. The latter is possible only when the nanoparticle is approximately perpendicular to the boundary. Thus The order parameter and concentration profiles of Janus particles of different length and different interaction strength are presented in Figs. 4-6. One can readily see that the orientational order parameter increases with the increasing interaction constant $\Delta J$ which specifies the selectivity of the Janus particle with respect to the monomers $\mathrm{A}$ and $\mathrm{B}$. The concentration of nanoparticles at the boundary also increases. It follows also from Figs. 5,6 that a moderate increase of the nanoparticle length does not effect these results dramatically. The width of the boundary region increases slightly and so does the orientational order parameter. The maxima of the order parameter profile are shifted away from the boundary and concentration of nanoparticles at the domain wall 
decreases because longer particles are distributed more evenly across the boundary layer.

\section{DISCUSSION}

In this paper we have considered the orientational ordering and spatial distribution of Janus nanoparticles in the lamellae phase of diblock copolymers using a molecular theory with fixed structure of block copolymer. It has been shown that Janus particles are predominantly located in the boundary region between the blocks and are strongly orientationally ordered. Janus nanoparticles are subject to the symmetric mean-field potential and as a result both concentration and order parameter profiles are symmetric with respect to the boundary. The concentration of nanoparticles possess a maximum directly at the boundary while the order parameter profiles possess two equal maxima shifted away from the boundary.

In contrast, symmetric nanoparticles are mainly located in one of the blocks where the interaction energy between a nanoparticle and the corresponding monomers is the lowest. Thus only a small fraction of all nanoparticles is orientationally ordered in the boundary region. Moreover, the symmetric nanoparticles are aligned parallel to the domain wall in one block and perpendicular to the wall in another block which results in the sign inversion of the orientational order parameter. Thus in this case the average anisotropy of the whole composite appears to be rather low.

The sufficiently large dielectric anisotropy of the nanocomposite is very important if one considers an attractive possibility to align block copolymers using the external electric field. One notes that alignment of block copolymers is a serious technological problem and no appropriate solution has been found so far. The coupling between the external electric field and the anisotropic medium is mainly determined by the average dielectric anisotropy. In a block copolymer, doped with anisotropic nanoparticles the macroscopic dielectric anisotropy is determined by the orientational order of the nanoparticles and by their concentration.. More precisely,the macroscopic anisotropy is approximately proportional to the following integral $\int \rho(z) S(z) d z$, where $\rho(z)$ is the local density of nanoparticles, $S(z)$ is the local nematic order parameter and the $z$-axis ir perpendicular to the lamellae planes. Thus thr block copolymers doped with symmetric nanoparticles are expected to possess a low dielectric anisotropy for the following two reasons. Firstly the concentration of symmtric 
nanopaerticls in the boundary region (where they are orientationally ordered) is rather low. Secondly, the orientational order parameter possess opposite signs on the sides of the domain wall which leads to a further decrease of the average anisotropy. In contrast, nanocomposites with Janus nanoparticles are expected to possess a sufficiently high anisotropy because such particles are mainly located in the boundary region and are strongly orientationally ordered with the same sign of the order parameter in all domains. Therefore one may conclude that polymer nanocomposites based on diblock copolymers doped with Janus nanoparticles are very promising anisotropic fluid materials which, in principle, can be aligned by moderates external fields. It would be very interesting to study such composites experimentally although, as far as we aware, they have not been synthesised yet..

\section{Acknowledgments}

The authors are grateful to the Russian Science Foundation (Project 16-13-10280) for financial support.

[1] Liquid Crystals with Nano and Microparticles, Lagerwall, J. P. F. and Scalia, G. (eds.). Singapore: World Scientific Publishing Company, (Series in Soft Condensed Matter; vol. 7)

[2] H. Qi, B. Kinkead, T. Hegmann,Effects of functionalized metal and semiconductor nanoparticles in nematic liquid crystal phases, Proc. SPIE 6911 (2008) 691106 (2008).

[3] H. Qi and T. Hegmann, "Formation of periodic stripe patterns in nematic liquid crystals doped with functionalized gold nanoparticles, J. Mater. Chem. 16 (2006) pp. 4197-4205.

[4] Y. Shiraishi, N. Toshima, K. Maeds, H. Yoshikawa, J. Xu, and S. Kobayashi:Frequency modulation response of a liquid-crystal electro-optic device doped with nanoparticles, Appl. Phys. Lett. 81 (2002) pp. 2845-2847.

[5] S.Kobayashi and N.Toshima,Nanoparticles and LCDs: It's a Surprising World, Information Display, 23 (2007) pp. 26-35.

[6] H. Yoshida, K. Kawamoto, H. Kubo, T. Tsuda, A. Fujii, S. Kuwabata, M. Ozaki,NanoparticleDispersed Liquid Crystals Fabricated by Sputter Doping, Advanced Materials 22 (2010) pp. $622-625$. 
[7] H.Yoshida, Y. Tanaka, K. Kawamoto, H. Kubo, T. Tsuda, A. Fujii, S. Kuwabata, H. Kikuchi, and M. Ozaki,Nanoparticle-Stabilized Cholesteric Blue Phases, Appl. Phys. Expr. 2 (2009) 121501.

[8] G. A. Shandryuk, E. V. Matukhina, R. B. Vasilev, A. Rebrov, G.N. Bondarenko, A.S. Merekalov, A.M Gas'kov, R.V. Tal'roze, Effect of H-Bonded Liquid Crystal Polymers on CdSe Quantum Dot Alignment within Nanocomposite, Macromolecules 41 (2008) pp. 2178-2185.

[9] R. V. Talroze, G. A. Shandryuk, A. S. Merekalov, A. M. Shatalova, and O. A. Otmakhova,Alignment of nanoparticles in polymer matrices, Polymer Science A, 51 (2009) pp. 1194-1202.

[10] P. Kopcansky, N. Tomasovicova, M. Koneracka, M. Timko, V. Zavisova, A. Dzarova, J. Jadzyn, E. Beaugnon, and X. Chaud, Phase Transitions in Liquid Crystal Doped with Magnetic Particles of Different Shapes, Int. J. Thermophys., 12 (2011) pp. 807-817.

[11] Yu.Reznikov, O.Buchnev, O.Tereshchenko, V.Reshetnyak, A.Glushchenko, and J.West, Ferroelectric Nematic Suspension, Appl. Phys. Lett.,82 (2003) pp. 1917-1919.

[12] F.Li, O.Buchnev, C.I. Cheon, A.Glushchenko, V.Reshetnyak, Yu.Reznikov, T.J.Sluckin, and J.L.West,Orientational Coupling Amplification in Ferroelectric Nematic Colloids, Phys.Rev.Lett. 97 (2006) 147801.

[13] M.V. Gorkunov and M.A. Osipov, Mean-field theory of a nematic liquid crystal doped with anisotropic nanoparticles, Soft Matter, 7 (2011) pp. 4348-4356.

[14] O. Buchnev, A. Dyadyusha, M. Kaczmarek, V. Reshetnyak, Yu. Reznikov, J. Opt. Soc. Am. B 24 (2007) 1512.

[15] E. Ouskova, O. Bunchev, V. Reshetnyak and Yu. Reznikov, Dielectric relaxation spectroscopy of a nematic liquid crystal doped with ferroelectric Sn2P 2S6 nanoparticles Liq.Cryst. 30 (2003) pp. 1235-1239).

[16] S. Kaur, S. P. Singh, A. M. Biradar, A. Choudhary, and K. Sreeniva,Analog switching in the nanocolloids of ferroelectric liquid crystals Appl. Phys. Lett. 91 (2007) 023120.

[17] A. Kumar, J. Prakash, D. S. Mehta, A. M. Biradar, and W. Haase, Analog switching in the nanocolloids of ferroelectric liquid crystals, Appl. Phys. Lett. 95 (2009) 023117.

[18] E. B. Barmatov, D. A. Pebalk, M. V. Barmatova,Influence of silver nanoparticles on the order parameter of liquid crystalline polymers, Liq. Cryst. 33 (2006) pp. 1059-1063.

[19] P. Kopansk, N. Tomaoviov, M. Konerack, M. Timko, Z. Mitrov, V. Zviov, N. ber, K. Fodor- 
Csorba, T. Tth-Katona, A. Vajda, J. Jadzyn, E. Beaugnon, X. Chaud,Structural phase transition in liquid crystal doped with gold nanoparticles, Acta Phys. Polonica A, 118 (2010) pp. 988-989.

[20] G. Sinha, C. Glorieux and J. Thoen, Broadband dielectric spectroscopy study of molecular dynamics in the glass-forming liquid crystal isopentylcyanobiphenyl dispersed with aerosils, Phys. Rev. E, 69 (2004) 031707.

[21] T. Bellini, M. Buscagli, C. Chiccoli, F. Mantegazza, P. Pasini and C. Zannoni,Nematics with Quenched Disorder: What Is Left when Long Range Order Is Disrupted? Phys. Rev. Lett., 85 (2000) 1008.

[22] H. Duran, B. Gazdecki, A. Yamashita and T. Kyu,Effect of carbon nanotubes on phase transitions of nematic liquid crystals, Liq. Cryst. 32 (2005) pp. 815-821.

[23] L.M.Lopatina and J.R.Selinger, Maier-Saupe-type theory of ferroelectric nanoparticles in nematic liquid crystals, Phys. Rev. E, 84 (2011) 041703.

[24] M. Alexandre, P. Dubois, Polymer-layered silicate nanocomposites: preparation, properties and uses of a new class of materials. Mater. Sci. Eng. Rev. 28, (2000) pp. 1-32.

[25] A. C. Balazs, Interactions of nanoscopic particles with phase-separating polymeric mixtures, Curr. Opin. Colloid Interface Sci. 4, (2000) pp. 443-448.

[26] E. P. Giannelis, Polymer-layered silicate nanocomposites: Synthesis, properties and applications, Appl. Organomet. Chem. 12, (1998) pp. 675-680.

[27] P. P. Soo, B. Y. Huang, Y. I. Jang, Y. M. Chiang, D. R. Sadoway, A. M. Mayes, Rubbery block copolymer electrolytes for solidstate rechargeable lithium batteries, J. Electrochem. Soc. 146, (1999) pp. 32-37.

[28] A. C. Balazs, T. Emrick, T. P. Russell, Nanoparticle polymer composites: where two small worlds meet, Science 314 (2006) pp. 1107-1110.

[29] R. Krishnamoorti, R. A. Vaia, Polymer Nanocomposites, J. Polym. Sci., Part B: Polym. Phys. 45, (2007) pp. 3252-3256.

[30] A. J. Crosby and J. Y. Lee, Polymer nanocomposites: the nano effect on mechanical properties, Polym. Rev. 47, (2007) pp.217-229.

[31] R.D. Deshmukh, Y. Liu, R.J. Composto,Two-Dimensional Confinement of Nanorods in Block Copolymer Domains, Nano Lett., 7 (2007) pp. 3662-3668.

[32] E. Ploshnik, A. Salant, U. Banin, R. Shenhar,Hierarchical Surface Patterns of Nanorods Ob- 
tained by Co-Assembly with Block Copolymers in Ultrathin Films, Adv. Mater.,22 ( 2010) pp. 2774-2779.

[33] E. Ploshnik, A. Salant, U. Banin, R. Shenhar,Co-assembly of block copolymers and nanorods in ultrathin films: effects of copolymer size and nanorod filling fraction, Phys. Chem. Chem. Phys., 12 (2010) pp. 11885-11893.

[34] K. Thorkelsson, A.J. Mastroianni, P. Ercius, T. Xu,Direct Nanorod Assembly Using Block Copolymer-Based Supramolecules, Nano Lett., 12 (2012) pp. 498-504.

[35] M.A. Osipov and M.V. Gorkunov,Spatial distribution and nematic ordering of anisotropic nanoparticles in lamellae and hexagonal phases of block copolymers, Eur. Phys. J. E, 39 (2016) pp. 126-134.

[36] A.V. Berezkin, Y.V. Kudryavtsev, M.V. Gorkunov, and M.A. Osipov, Ordering of anisotropic nanoparticles in diblock copolymer lamellae: Simulations with dissipative particle dynamics and a molecular theory, J. Chem. Phys., 146 (2017) 144902 .

[37] M. A. Osipov, A. S. Ushakova, M. V. Gorkunov, Orientational ordering of nanorods in diblock copolymers, Liquid Crystals, (2017) DOI: 10.1080/02678292.2017.1346826 . 


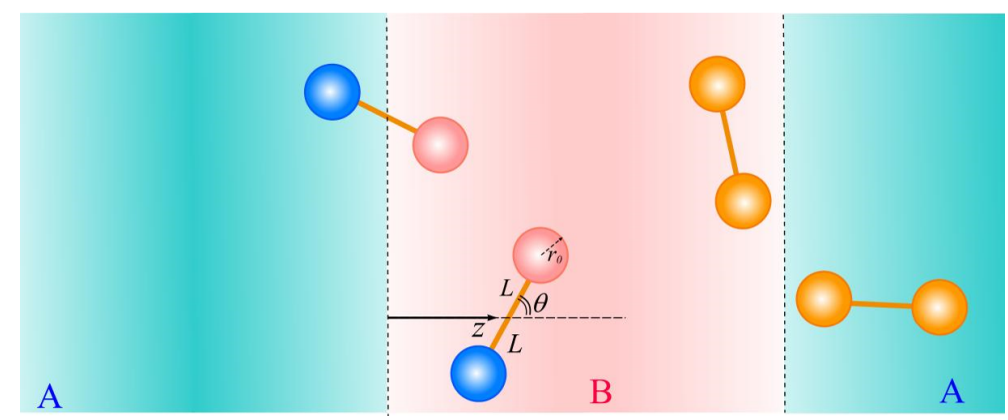

FIG. 1: Alignment of Janus and symmetric nanoparticles with two interaction centres in the boundary region between adjacent blocks. One interaction centre of the Janus nanoparticle has affinity to the block A while another centre has affinity to the block B. Janus particles are aligned approximately perpendicular to the boundary while symmetric particels are aligned parallel to the boundary in one block and perpendicular to the boundary in another one.

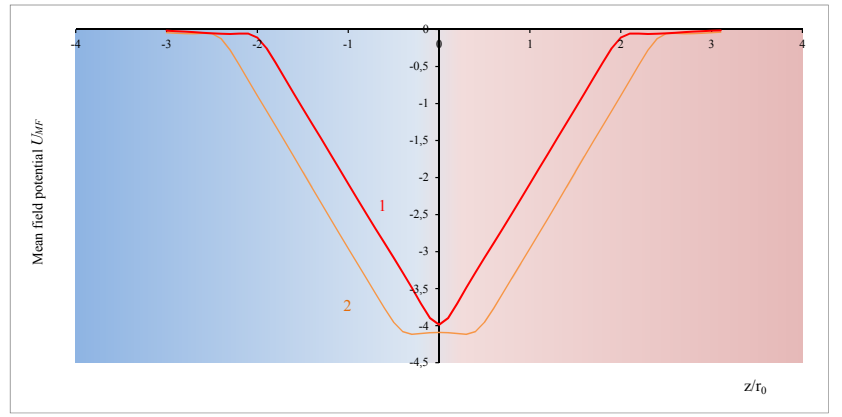

FIG. 2: The mean field potential of a Janus nanoparticle with two interaction centres as a function of the distance $z$ between the nanoparticle centre and the flat boundary for $L=2 r_{0}$ and for two different values of the angle $\theta$ between the nanoparticle and the boundary normal : $\theta=\pi / 3$ (1) and $\theta=\pi / 4(2)$. 


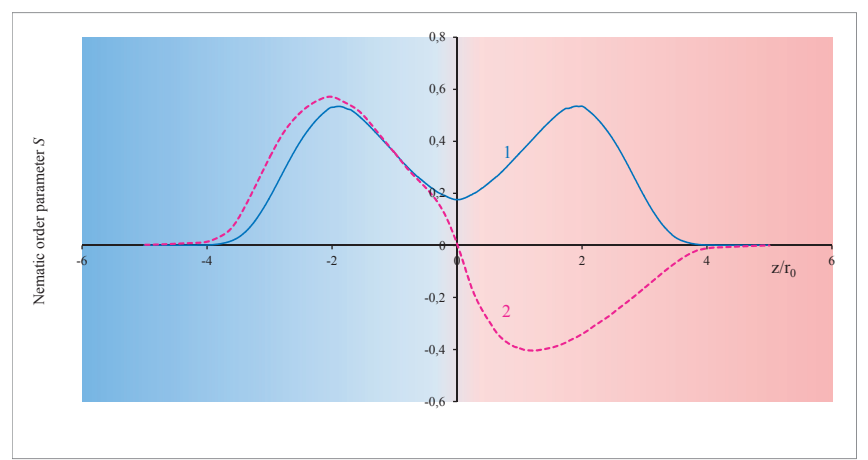

FIG. 3: Orientational order parameter profiles of Janus nanoparticles (1) and symmetric nanoparticles (2) in the boundary region between the two blocks in the lamellae phase of the diblock copolymer. The interaction constant $\Delta J=k_{B} T$ and $L=r_{0}$.

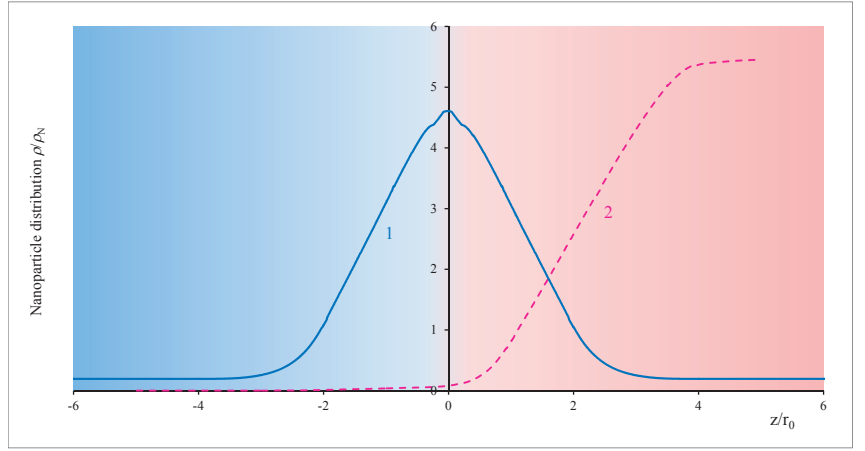

FIG. 4: Concentration profiles of Janus nanoparticles (1) and symmetric nanoparticles (2) in the lamellae phase of the diblock copolymer. The interaction constant $\Delta J=k_{B} T$ and $L=r_{0}$. 


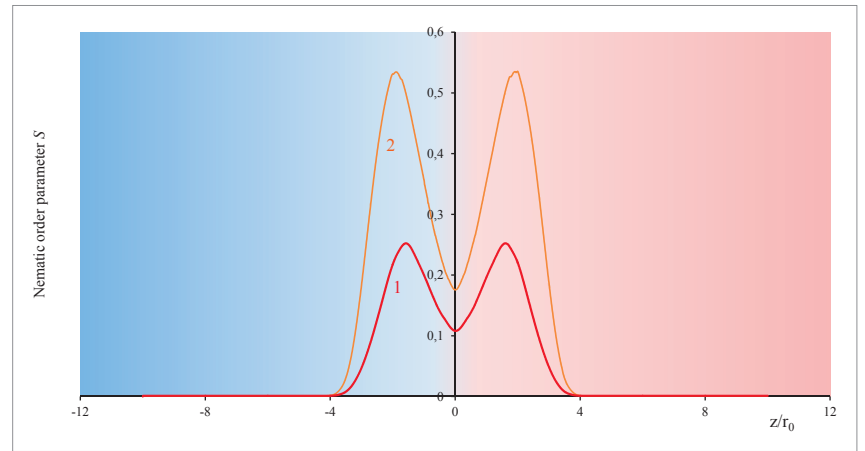

FIG. 5: Orientational order parameter profiles of short Janus nanoparticles with $L=r_{0}$ in th boundary region between the two blocks in the lamellae phase of the diblock copolymer for two different values of the interaction constant $\Delta J=k_{B} T$ (1) and $\Delta J=2 k_{B} T$ (1).

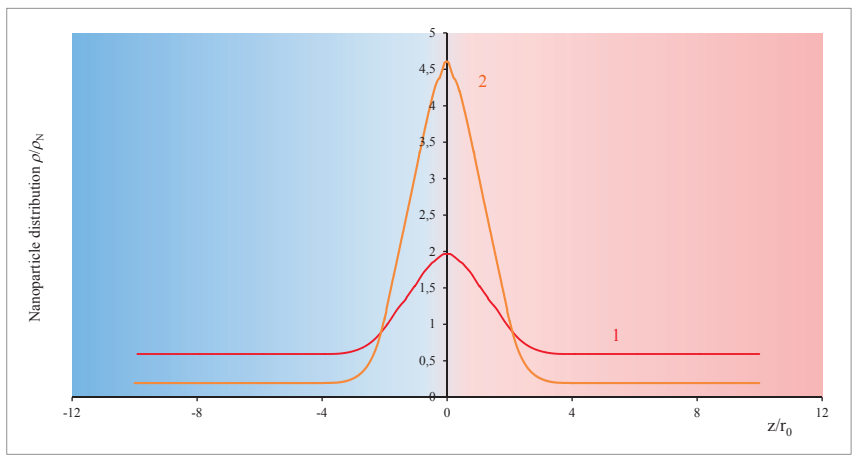

FIG. 6: Local concentration of short Janus nanoparticles with $L=r_{0}$ in the lamellae phase of the diblock copolymer for two different values of the interaction constant $\Delta J=k_{B} T$ (1) and $\Delta J=2 k_{B} T(1)$. 


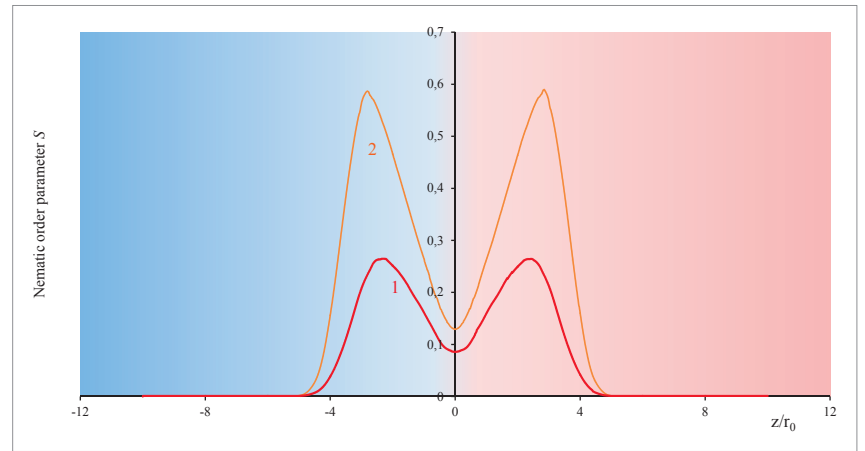

FIG. 7: Orientational order parameter profiles of long Janus nanoparticles with $L=2 r_{0}$ in th boundary region between the two blocks in the lamellae phase of the diblock copolymer for two different values of the interaction constant $\Delta J=k_{B} T$ (1) and $\Delta J=2 k_{B} T$ (1).

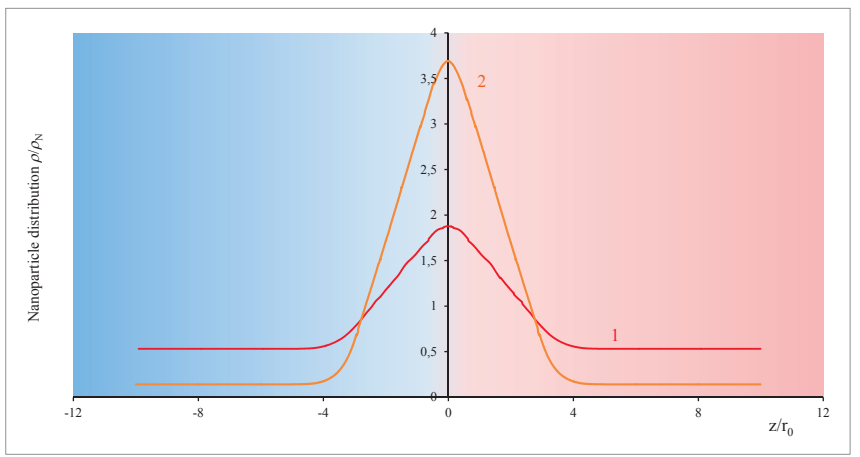

FIG. 8: Local concentration of long Janus nanoparticles with $L=2 r_{0}$ in the lamellae phase of the diblock copolymer for two different values of the interaction constant $\Delta J=k_{B} T$ (1) and $\Delta J=2 k_{B} T(1)$. 